\title{
Improving HF Radar Estimates of Surface Currents Using Signal Quality Metrics, with Application to the MVCO High-Resolution Radar System
}

\author{
ANTHONY R. KIRINCICH \\ Woods Hole Oceanographic Institution, Woods Hole, Massachusetts \\ TONY DE PAOLO AND ERIC TERRILL \\ Scripps Institution of Oceanography, La Jolla, California
}

(Manuscript received 16 September 2011, in final form 1 May 2012)

\begin{abstract}
Estimates of surface currents over the continental shelf are now regularly made using high-frequency radar (HFR) systems along much of the U.S. coastline. The recently deployed HFR system at the Martha's Vineyard Coastal Observatory (MVCO) is a unique addition to these systems, focusing on high spatial resolution over a relatively small coastal ocean domain with high accuracy. However, initial results from the system showed sizable errors and biased estimates of $M_{2}$ tidal currents, prompting an examination of new methods to improve the quality of radar-based velocity data. The analysis described here utilizes the radial metric output of CODAR Ocean Systems' version 7 release of the SeaSonde Radial Site Software Suite to examine both the characteristics of the received signal and the output of the direction-finding algorithm to provide data quality controls on the estimated radial currents that are independent of the estimated velocity. Additionally, the effect of weighting spatial averages of radials falling within the same range and azimuthal bin is examined to account for differences in signal quality. Applied to two month-long datasets from the MVCO high-resolution system, these new methods are found to improve the rms difference comparisons with in situ current measurements by up to $2 \mathrm{~cm} \mathrm{~s}^{-1}$, as well as reduce or eliminate observed biases of tidal ellipses estimated using standard methods.
\end{abstract}

\section{Introduction}

Over the past two-and-a-half decades, numerous efforts have been made to understand the errors and biases that exist for high-frequency radar (HFR) observations of ocean surface currents. This is especially true for the direction-finding calculation that is a major part of extracting surface currents from the SeaSondetype radar systems examined here, made by CODAR Ocean Systems. While these efforts have allowed progress in understanding the roles of antenna-based bearing biases, direction-finding algorithm performance, and velocity-based quality control schemes, the role of external data quality indicators (i.e., those independent of the estimated velocity itself) have received less attention. This work intends to show that using nonvelocity-based

Corresponding author address: Anthony Kirincich, Woods Hole Oceanographic Institution, 266 Woods Hole Road, Woods Hole, MA 02543.

E-mail: akirincich@whoi.edu metrics of the signal quality and the direction-of-arrival (DOA) function to both implement additional data quality controls and alter the typical spatial averaging process can lead to surface currents with reduced scatter and biases when compared to in situ current sensors.

This effort is motivated by the installation of a new system of closely spaced HF radars deployed along the southern coast of Martha's Vineyard, Massachusetts. Initial comparisons between HFR surface currents and in situ observations of near-surface velocity from an acoustic Doppler current profiler (ADCP) revealed large scatter of hourly near-surface velocities and, more importantly, significant biases in estimates of the $M_{2}$ tidal ellipses (the dominant tidal constituent). These biases are likely the result of systematic errors in the radar estimates of surface currents. Because the purpose of this high-resolution system is to observe the spatially variable inner-shelf circulation at a variety of scales, the increased spatial independence and accuracy needs of the science goals, coupled with these initial results, 
(A) Range/velocity spectra from 3 receive antennas ensemble averaged

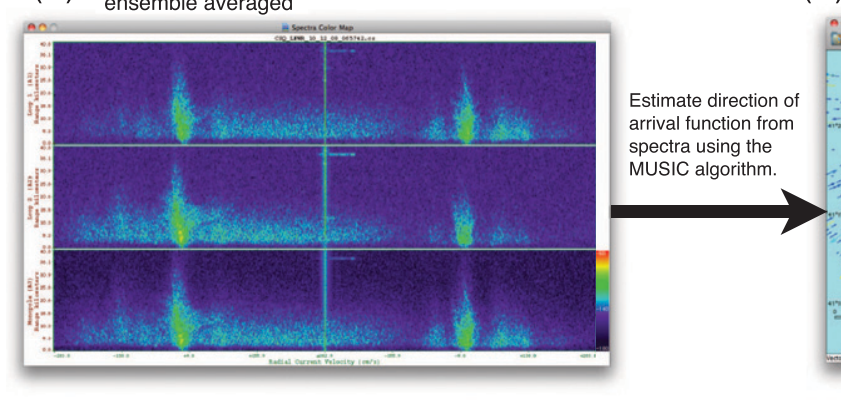

(B) Radial metrics output

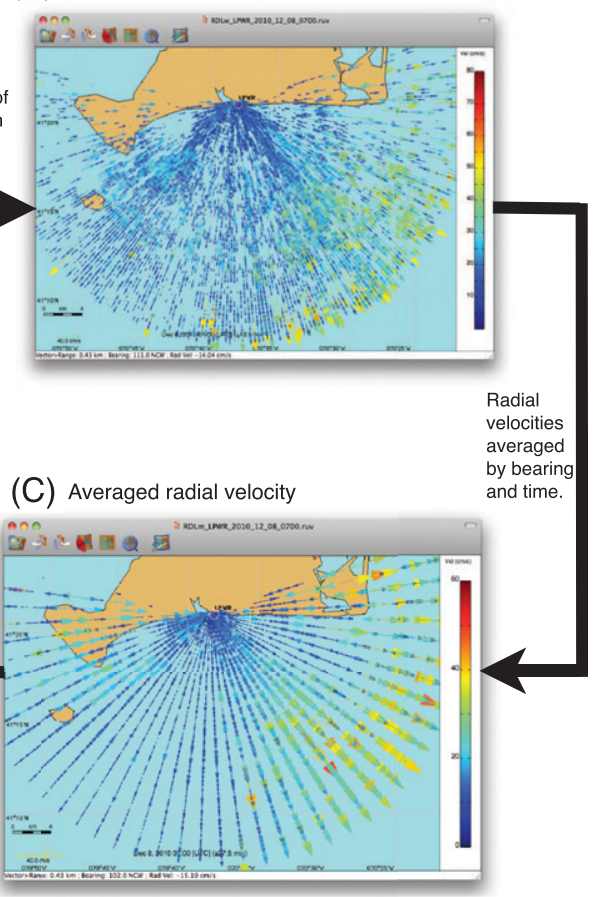

FIG. 1. (a)-(d) Illustrating the major processing steps to derive estimates of vector surface currents from the backscatter obtained by direction-finding SeaSonde HF radar systems. All images were taken from the COS Radial and Combine Site Software packages.

necessitated an examination of new methods to improve data quality.

This paper is organized as follows. A summary of HFR data acquisition, typical data-processing methods, and recent work on understanding and/or minimizing HFR velocity errors for the CODAR Ocean Systems' SeaSonde radar is presented first, both for completeness and to give context to the changes examined here. The new HFR system located at the Martha's Vineyard Coastal Observatory (MVCO) is described next along with the initial results of the examined datasets. Improvements to the data quality controls and averaging procedures are then described using data from the MVCO system. Finally, the results of these steps applied to two separate MVCO HFR datasets are shown and conclusions are presented.

\section{SeaSonde data acquisition and quality \\ a. Data acquisition}

HF radars measure ocean surface velocities by emitting a vertically polarized electromagnetic signal toward the electrically conductive ocean surface. The outgoing signal is coherently backscattered by surface gravity waves whose wavelengths are half that of the transmitted signal wavelength (i.e., 6-m ocean wavelengths for $24-27-\mathrm{MHz}$ systems) and travel directly away from or toward the transmitter. This coherent, strong return of energy at a precise wavelength (Bragg scattering) allows the radial velocity (away from the receiver) of the surface water to be inferred from the Doppler shift of the returning signal (Crombie 1955; Barrick and Weber 1977; Paduan and Graber 1997). For the compact SeaSonde HFR system, manufactured by CODAR Ocean Systems, a series of three collocated receive antennas, mounted orthogonally, are used along with a direction-finding (DF) algorithm to infer the bearing of the incoming radar return (Barrick and Lipa 1997). A series of temporal and spatial averaging and interpolation steps are employed before and after direction finding to grid the results to a regularly spaced (in range and azimuth) grid of radial velocities. Vector velocities are then obtained using the results from multiple sites.

For DF systems like the SeaSonde, four distinct processing steps exist (Fig. 1). First, from each of the three receive antennas located at a site, a cross-spectral estimate of the complex signal power is produced every sample period (nominally either 4 or $8 \mathrm{~min}$ ), which represents the reflected energy at each detectable range distance and Doppler frequency (Fig. 1a). Because Doppler frequency shifts can be translated directly to 
“Doppler velocities," these spectra provide estimates of velocities observed along each range circle. Successive spectra for each range circle are normally averaged over a time interval (e.g., a 30-min average every $10 \mathrm{~min}$ or a 1-h average every $1 / 2 h$ ) to reduce noise and attain an ensemble average of the complex signal voltages at each Doppler velocity. Second, from these averaged range circle spectral estimates, for each measured Doppler velocity within the region of the strongest returns (the "firstorder region"), the complex signal voltages from each of the three antennas are used with a direction-finding algorithm [Multiple Signal Classification (MUSIC); see Schmidt (1986)] to find the DOA function for each observed velocity (Lipa et al. 2006). The peak value(s) of the DOA function give the estimated bearing of the signal(s), with a maximum of two bearings allowed by the threeantenna array. With each measured Doppler velocity reorganized by estimated bearing (Fig. 1b), all of the velocity estimates at a given range having bearings within a nominally $5^{\circ}$-wide azimuthal angle are averaged to estimate the radial velocity (e.g., every $1 / 2 \mathrm{~h}$ over an hour or every $1 \mathrm{~h}$ over $3 \mathrm{~h}$ ) in each range/azimuthal bin (Fig. 1c). These final averaged radial estimates are used, along with the results from other sites, to estimate the vector velocities present on a Cartesian grid using a spatial averaging window typically $1.5-2$ times larger than the grid spacing itself (COS 2009; Fig. 1d). In addition to the multiple layers of averaging described above, interpolation is typically used to subsample the spectra for finer velocity resolution and, on both the radial and vector velocities, to fill gaps in the coverage area and spatially smooth the resulting currents (Lipa et al. 2006).

\section{b. Data quality}

Multiple comparative studies have been made between HFR data and near-surface measurements of velocity using ADCPs or Lagrangian drifter trajectories to assess the quality of the surface current observations (Emery et al. 2004; Ullman and Codiga 2004; Kohut et al. 2006; Paduan et al. 2006; Ohlmann et al. 2007). In theory, velocity errors, as rms differences with in situ observations, can vary dramatically due to transmission frequency, sensor placement, and location within the sampled domain. However, in practice, these works have shown that an upper bound of $10-20 \mathrm{~cm} \mathrm{~s}^{-1}$ exists for long-range (4-5 MHz) systems with slightly reduced values for 11-13- or 24-26-MHz systems. Noise levels of $6 \mathrm{~cm} \mathrm{~s}^{-1}$ have been inferred from these comparisons (Emery et al. 2004; Ohlmann et al. 2007).

Additional efforts have focused on the potential sources of error in HFR observations, finding that differences between HFR and in situ observations can be due to a combination of poor antenna calibrations, adverse environmental conditions, and either poor or variable performance of the direction-finding algorithm. Emery et al. (2004) compared radial velocities from bearings of up to $\pm 30^{\circ}$ away from the true bearing to ADCP-based near-surface velocity along the true bearing, finding that bearing errors of up to $10^{\circ}-15^{\circ}$ routinely existed when the ideal beam patterns were used. Use of the measured or calibrated antenna response patterns can minimize these types of errors (Barrick and Lipa 1986; Kohut and Glenn 2003); however, determining the true antenna response pattern itself is somewhat subjective, because different interpretations of the calibration data can lead to significantly different azimuthal distributions of the radial velocities (Cosoli et al. 2010). Examining the role of environmental effects on perceived errors between sensors, Ohlmann et al. (2007) found that the rms differences between HFR radial velocities from a single radial cell and ensembles of drifter velocities obtained within the cell were similar to rms differences between the drifter velocities themselves. Thus, environmental conditions can have a significant effect on these standard rms difference comparisons. Under nearly ideal environmental and operational conditions, Kohut et al. (2006) found estimated differences as low as $3 \mathrm{~cm} \mathrm{~s}^{-1}$ for a well-averaged, 25-MHz system.

A smaller number of works have examined the output of the DF algorithm and both the effects of noise on algorithm performance and the parameters used to differentiate when a single- or dual-angle solution is appropriate. Simulations of the effect of the DF algorithm on velocity error with synthetic datasets have indicated that, under ideal antenna conditions and an ensemble of various simulated flow conditions, a lower bound of $3 \mathrm{~cm} \mathrm{~s}^{-1}$ error exists solely due to the direction-finding algorithm (Laws et al. 2010). Using synthetic datasets from four specific current scenarios, de Paolo and Terrill (2007a,b) found that the skill of the MUSIC algorithm decreased significantly when signal-to-noise ratios (SNRs) became less than 10 . Further, during the more complex current scenarios examined, adjusting the dual-angle solution parameters used in the HFR implementation of MUSIC (Lipa et al. 2006) led to a higher fraction of dualangle solutions, but not necessarily lower rms differences (de Paolo and Terrill 2007a).

Despite the considerable number of comparative studies and error investigations performed to date, the application of these results to advance HFR data quality control have been more limited. While many, if not all, studies utilizing HFR data implement some level of data quality controls (e.g., Kim et al. 2007), most focus on using the estimated velocities themselves to gauge the quality of the radial or vector velocities, following standard time series analysis techniques (e.g., NDBC 2009; 
Halle 2008). In addition, error estimates reported by the SeaSonde instrument software are composed of a simple standard deviation of all velocities mapped into the given range/azimuthal bin by the DF algorithm (COS 2009). Only two previous studies (de Paolo and Terrill $2007 a, b$ ) have examined the signal quality or the output of MUSIC, metrics other than the velocities themselves, as a way to quality control the velocity results.

The present analysis builds on that used by de Paolo and Terrill (2007a,b), utilizing the radial metric output of CODAR Ocean Systems' version 7 release of the SeaSonde Radial Site Software Suite to examine both the parameters of the received signals and the DF algorithm processing to provide and utilize quality control metrics for the estimated radial currents. Additionally, this work examines a new way to selectively average radials falling within the same cell in order to account for differences in signal quality and velocity error. Applied to the MVCO high-resolution HFR system, these new efforts are found to both improve the rms difference comparisons with in situ instruments and, more importantly, reduce observed biases of tidal ellipses estimated using the MVCO HFR system.

\section{The MVCO HFR system}

MVCO, located along the south coast of the island of Martha's Vineyard (Fig. 2), supports a new HFR system designed to map inner-shelf currents with the highest possible spatial resolution. Deployed in 2010, the system has the technical goal of measuring currents at scales approaching $400 \mathrm{~m}$ within a $20 \mathrm{~km} \times 20 \mathrm{~km}$ domain south of Martha's Vineyard (Fig. 2). The system is composed of three closely spaced sites with SeaSonde-type DF instruments running at operating frequencies near $25 \mathrm{MHz}$. Two of the three sites are located on land, with one placed at the MVCO Shore Meteorological Station (METS) and the second approximately $10 \mathrm{~km}$ to the west at the Long Point Wildlife Refuge (LPWR). The third site is located on the MVCO Air-Sea Interaction Tower (ASIT), approximately $4 \mathrm{~km}$ offshore and south of the island. To achieve the highest possible radial resolution $(420 \mathrm{~m})$ given the $350 \mathrm{kHz}$ of bandwidth available at $25 \mathrm{MHz}$, all sites run at common frequencies using GPSbased timing to separate the transmissions from each site (Table 1). Given the small spatial domain that can be adequately sampled at low geometric error with this configuration, as well as potential for interactions between the instruments at the land and tower sites, all sites transmit at low power (1-2 W, less than 3\% of typical systems). In comparison, most coastal, nonestuarian HFR sites operate at 50-60 W and obtain radial resolutions of $2-8 \mathrm{~km}$ out to ranges of approximately $60-130 \mathrm{~km}$,

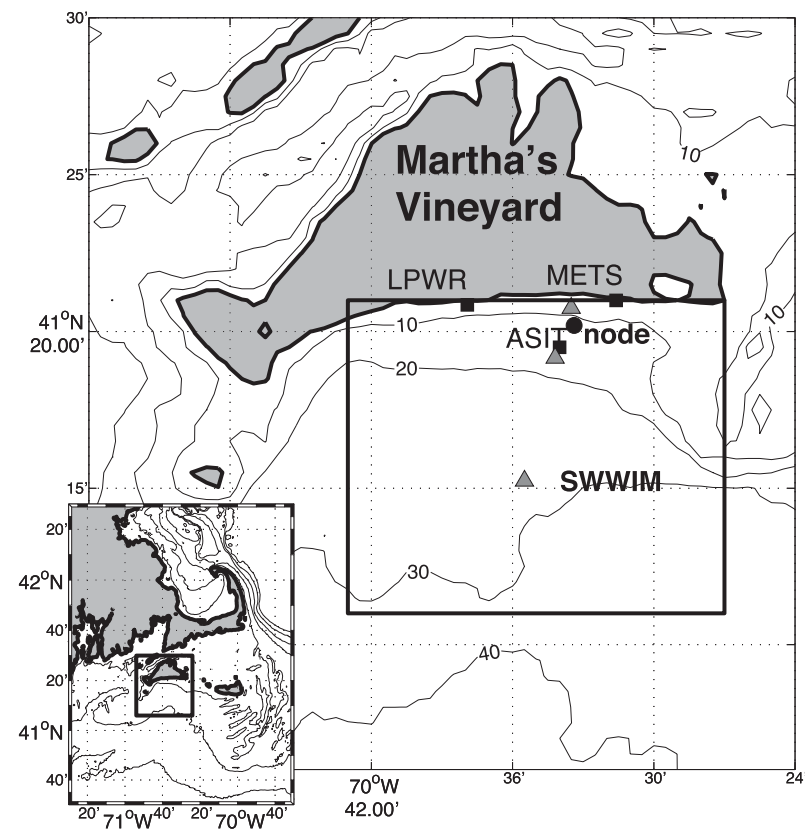

FIG. 2. Map of Martha's Vineyard. The land-based meteorological mast and offshore tower installations of the MVCO are shown (METS and ASIT), as is the location of the MVCO 12-m underwater node (node), which provided ADCP observations for the study. MVCO radar sites are located at METS, ASIT, and LPWR, and the sampling domain shown in Fig. 3 is shown (black box). The locations of the previous observational sites (Lentz et al. 2008), used for additional comparisons, are marked (triangles). Bathymetric contours are shown (m).

depending on which operating frequency is used, 24-26, 11-13, or 4-5 MHz.

To obtain their science goals, the MVCO HFRs were configured to maximize the spatial and temporal independence of the observations. Spectral estimates of the observed Doppler-shifted velocities are collected in bursts of 1028 nonoverlapping frequency sweeps with a sweep rate of $2 \mathrm{~Hz}$ for finer-resolution Doppler velocities than is typical for $25-\mathrm{MHz}$ systems without interpolation. A maximum of three, but normally two, successive spectral estimates are averaged to create the necessary ensemble estimate every $15 \mathrm{~min}$. Direction finding and azimuthal averaging into $5^{\circ}$ bands is performed on each ensemble and, for data processed using the standard software suite, successive radial velocity estimates are time averaged into 60-min averages every $30 \mathrm{~min}$. No interpolation is used to smooth the fields or fill in radial gaps, but, as in previous works, outliers are removed before computing vector velocities. Given the dense spacing of the radial grid points (Fig. 3), the vector averaging is performed using a $400-\mathrm{m}$ grid with grid points starting approximately $600 \mathrm{~m}$ offshore and an averaging radius equal to the grid width. These alternative methods were used to achieve finer-resolution velocity 
TABLE 1. MVCO HF radar operating parameters.

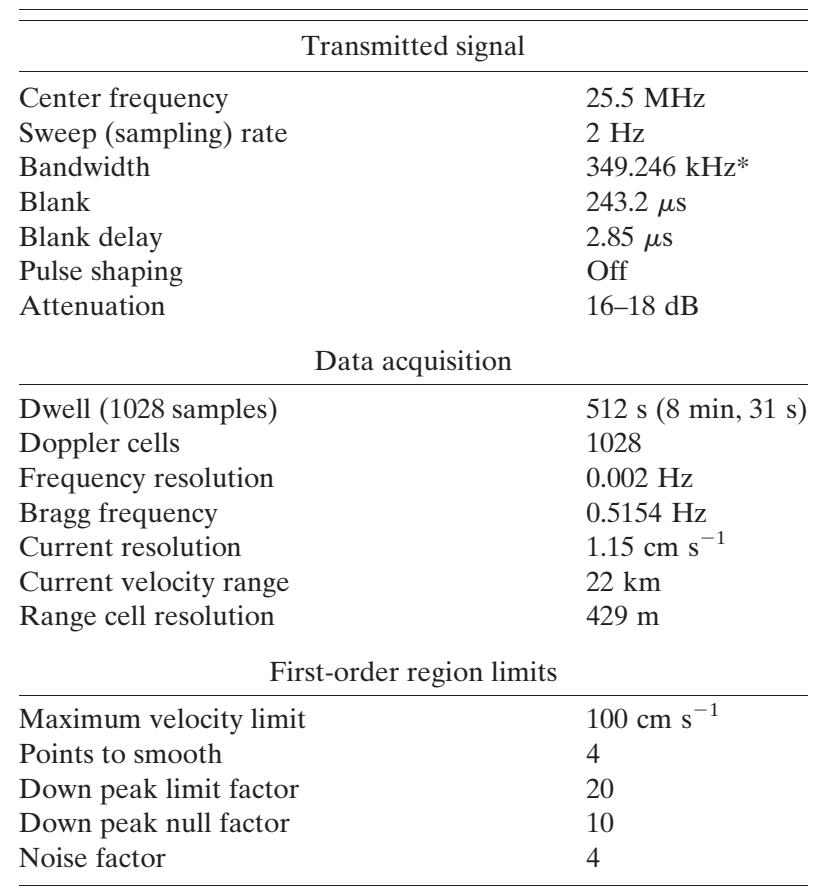

* Small frequency offsets and different GPS-based timing alignments are used to minimize interference between the sites.

estimates having greater spatial independence at the potential expense of increased noise.

For the datasets used here, a number of steps were taken to ensure that the radial velocity estimates were of the highest quality possible. The first-order region limits utilized for the analysis, optimized for the conditions present south of Martha's Vineyard, are shown in Table 1. Measured antenna patterns were obtained for each site and utilized in the DF algorithm to estimate radial currents over water. Finally, the spatial structure of the $M_{2}$ tidal ellipses for the entire domain, estimated from the vector velocity time series at each grid point using T_Tide (Pawlowicz et al. 2002), were analyzed for patterns of unrealistic ellipse inclination (orientation) emanating from a particular site, which serve as an indication of potential bearing-related errors. The measured beam patterns were adjusted by smoothing and/or interpolation, similar to that described by Cosoli et al. (2010) and de Paolo and Terrill (2007a), to minimize errors identified.

\section{Initial results using standard methods}

To test the representativeness of the surface velocities obtained by the MVCO HFR system, the surface current estimates (as hourly averages) were compared to observations of near-surface velocities measured by a bottom-mounted, upward-looking RD Instruments

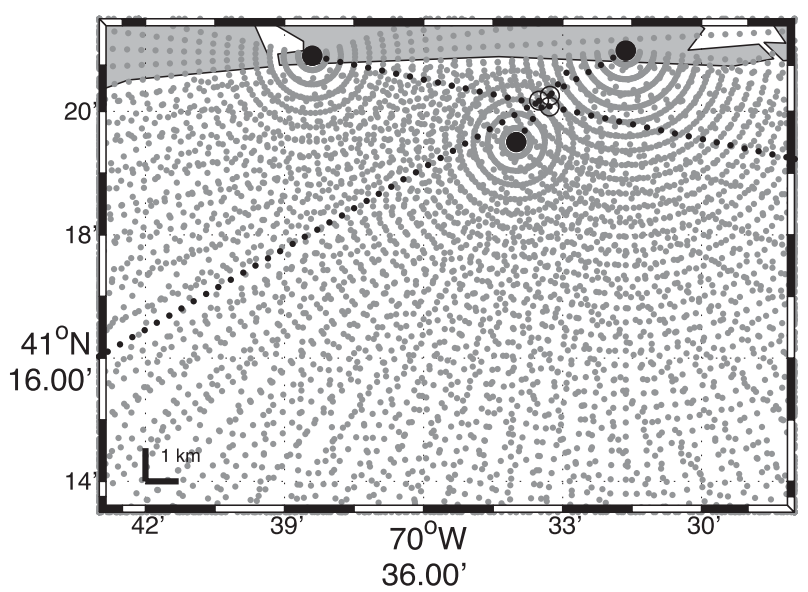

FIG. 3. Sampling domain of the MVCO radar system. The estimated position of each radial average from all stations are shown (gray dots), along with the specific radial lines (black dots) and cells (open circles) that overlay the 12-m-node ADCP location.

$1200-\mathrm{kHz}$ ADCP at the MVCO underwater node. The underwater node is located $1.6 \mathrm{~km}$ offshore in $12 \mathrm{~m}$ of water and sampled using $0.5-\mathrm{m}$ bin depths. Velocity observations from the ADCP were masked using a tidefollowing surface level minus $1.5 \mathrm{~m}$, to account for sidelobe contamination, and then linearly extrapolated back to the theoretical effective radar depth of $0.25 \mathrm{~m}$ (Stewart and Joy 1974) using the top three viable bins of the ADCP. While this surface extrapolation is used in the comparisons given below, it is important to note that qualitatively similar results were found using observations from the highest bin of the ADCP, indicating that the extrapolation of ADCP velocities to the surface did not significantly affect the results presented. For radial comparisons, the ADCP vector velocities were rotated into the along- and across-bearing coordinate system of each of the sites. A harmonic analysis of the tidal velocities present was performed on both ADCP and HFR velocities using T_Tide (Pawlowicz et al. 2002).

A key difference between the surface currents obtained by the HFR system and those observed by the ADCP is that the Lagrangian Stokes drift caused by surface gravity waves are present in the HFR surface currents, but not in the Eularian ADCP velocities. Theoretical work by Creamer et al. (1989) indicated that radar surface currents should include the Stokes component of all surface gravity waves with wavelengths larger that the Bragg wave $(6 \mathrm{~m}$ for $25 \mathrm{MHz}$ ). Recent observational studies by Mao and Heron (2008) and Ullman et al. (2006) have somewhat confirmed this, showing that model fits to the surface currents using the wind (Mao and Heron 2008), as well as comparisons between drifter and radar observations from different effective depths (Ullman et al. 2006), were significantly improved if the effects of Stokes drift were 
TABLE 2. MVCO 12-m node ADCP near-surface tidal constituents.

\begin{tabular}{|c|c|c|c|c|c|c|}
\hline Constituent & Period (h) & Dataset & Semimajor axis $\left(\mathrm{cm} \mathrm{s}^{-1}\right)$ & Semiminor axis $\left(\mathrm{cm} \mathrm{s}^{-1}\right)$ & Inclination $\left({ }^{\circ}\right)$ & Phase $\left(^{\circ}\right)$ \\
\hline \multirow[t]{2}{*}{$M_{2}$} & 12.42 & Fall & $25.6 \pm 4.1$ & $-4.0 \pm 0.9$ & $179 \pm 3$ & $200 \pm 10$ \\
\hline & & Winter & $24.5 \pm 1.4$ & $-3.0 \pm 0.5$ & $1.4 \pm 1.5$ & $178 \pm 4$ \\
\hline \multirow[t]{2}{*}{$N_{2}$} & 12.65 & Fall* & & & & \\
\hline & & Winter & $4.1 \pm 1.5$ & $-0.9 \pm 0.6$ & $176 \pm 11$ & $163 \pm 2$ \\
\hline \multirow[t]{2}{*}{$S_{2}$} & 12.00 & Fall & $3.6 \pm 3.8$ & $-0.8 \pm 1.2$ & $168 \pm 19$ & $249 \pm 67$ \\
\hline & & Winter & $2.9 \pm 1.6$ & $-0.5 \pm 0.6$ & $170 \pm 12$ & $217 \pm 35$ \\
\hline \multirow[t]{2}{*}{$O_{1}$} & 25.82 & Fall & $2.4 \pm 2.5$ & $0.2 \pm 1.3$ & $0 \pm 31$ & $59 \pm 66$ \\
\hline & & Winter & $1.3 \pm 1.0$ & $-0.2 \pm 0.4$ & $178 \pm 20$ & $260 \pm 49$ \\
\hline \multirow[t]{2}{*}{$K_{1}$} & 23.93 & Fall & $0.5 \pm 1.7$ & $0.1 \pm 1.2$ & $118 \pm 89$ & $284 \pm 180$ \\
\hline & & Winter & $2.7 \pm 1.0$ & $-0.2 \pm 0.4$ & $177 \pm 9$ & $312 \pm 24$ \\
\hline
\end{tabular}

* The fall sample period was not long enough to resolve the $N_{2}$ constituent.

included. Thus, to aid the comparisons made here, a time series of the total Stokes drift at the effective depth of the radar observations was subtracted from the HFR surface current time series. ADCP-based estimates of the significant wave height, dominant wave period, and significant wave direction, calculated by MVCO using standard analysis methods and the 12-m node ADCP observations, were used to estimate the Stokes drift present at the radar's effective depth using linear wave theory following Lentz et al. (2008). Tests with and without the estimate of Stokes drift subtracted from the HFR surface currents indicated that not accounting for Stokes drift increased the rms differences given below by $1-1.5 \mathrm{~cm} \mathrm{~s}^{-1}$.

Two initial datasets of surface currents from the array were examined to understand system performance using two or three sites. The "fall" dataset covers the 4-week period from 17 September to 14 October 2010 and is comprised of radial and vector currents from the two land-based sites only, because the ASIT site had not yet been installed. The "winter" dataset covers a slightly longer period between 5 December 2010 and 13 January 2011 when data from all three sites were available. Based on observations from the ADCP, mean surface velocities were $3 \mathrm{~cm} \mathrm{~s}^{-1}$ to the west and $2.5 \mathrm{~cm} \mathrm{~s}^{-1}$ to the south during the fall period and 0.4 and $1.3 \mathrm{~cm} \mathrm{~s}^{-1}$ to the west and south during the winter period, respectively. Tidal velocities were the strongest component of flow during both datasets, being an order of magnitude greater than the estimated mean velocities (Table 2). While the magnitude of the dominant $M_{2}$ tidal constituent of the depth-averaged mean ADCP velocities (not shown here) was similar between the datasets, small but significant differences existed in the estimated semimajor and semiminor axes magnitudes of the near-surface velocities of the two datasets (Table 2). The near-surface velocities of the fall dataset, occurring near or at the end of the summer-stratified period, were marked by weak wind forcing and slightly stronger tidal currents. Mean flow during this time period appears to be driven by tidal rectification (Ganju et al. 2011). Conditions during the winter dataset were typical for that time of year, with frequent strong wind events, strong surface currents, and much weaker stratification as well as slightly weaker surface tidal currents.

Error estimates, as root-mean-square (rms) differences between the near-surface ADCP velocities and HFR radial or vector surface currents of the MVCO system, were near the higher end of published estimates of errors for midrange HFR measurements. For the fall dataset, rms differences between the radial currents at LPWR and METS and the node velocities were 10.4 and $9.4 \mathrm{~cm} \mathrm{~s}^{-1}$, respectively (Fig. 4). Comparisons of vector surface currents of the fall dataset had rms differences of 7.7 and $10.3 \mathrm{~cm} \mathrm{~s}^{-1}$ for the east and north velocities, respectively. Errors for the ADCP HFR comparisons during the winter dataset were similar to the fall dataset for the individual radial estimates but were reduced for the vector surface currents. RMS differences ranged from 7.9 to $9.6 \mathrm{~cm} \mathrm{~s}^{-1}$ for the radial velocities at all three sites, and 6.3 and $6.9 \mathrm{~cm} \mathrm{~s}^{-1}$ for the east and north velocities (Fig. 5). Time series of radial currents from similar ranges but varying bearing angles were also compared to the ADCP velocities to test for antenna-based bearing errors following Emery et al. (2004). At all sites, the highest correlations and lowest rms differences occurred at the bearing angle of the MVCO node, indicating that minimal residual bearing errors existed for radial currents at the location of the ADCP.

Comparisons of the estimated $M_{2}$ tidal components of the ADCP and nearby HFR surface currents enable the identification of systematic biases that random noise might obscure in comparisons of raw hourly averaged velocities. The $M_{2}$ tide is a narrowbanded signal with a wide spatial extent; thus, each instrument should be able to capture it robustly to accuracies much greater than those of raw or hourly averaged velocities. In general, comparisons between the $M_{2}$ components of the radial or vector velocities had much lower rms differences than those described above. During the fall dataset, estimates 

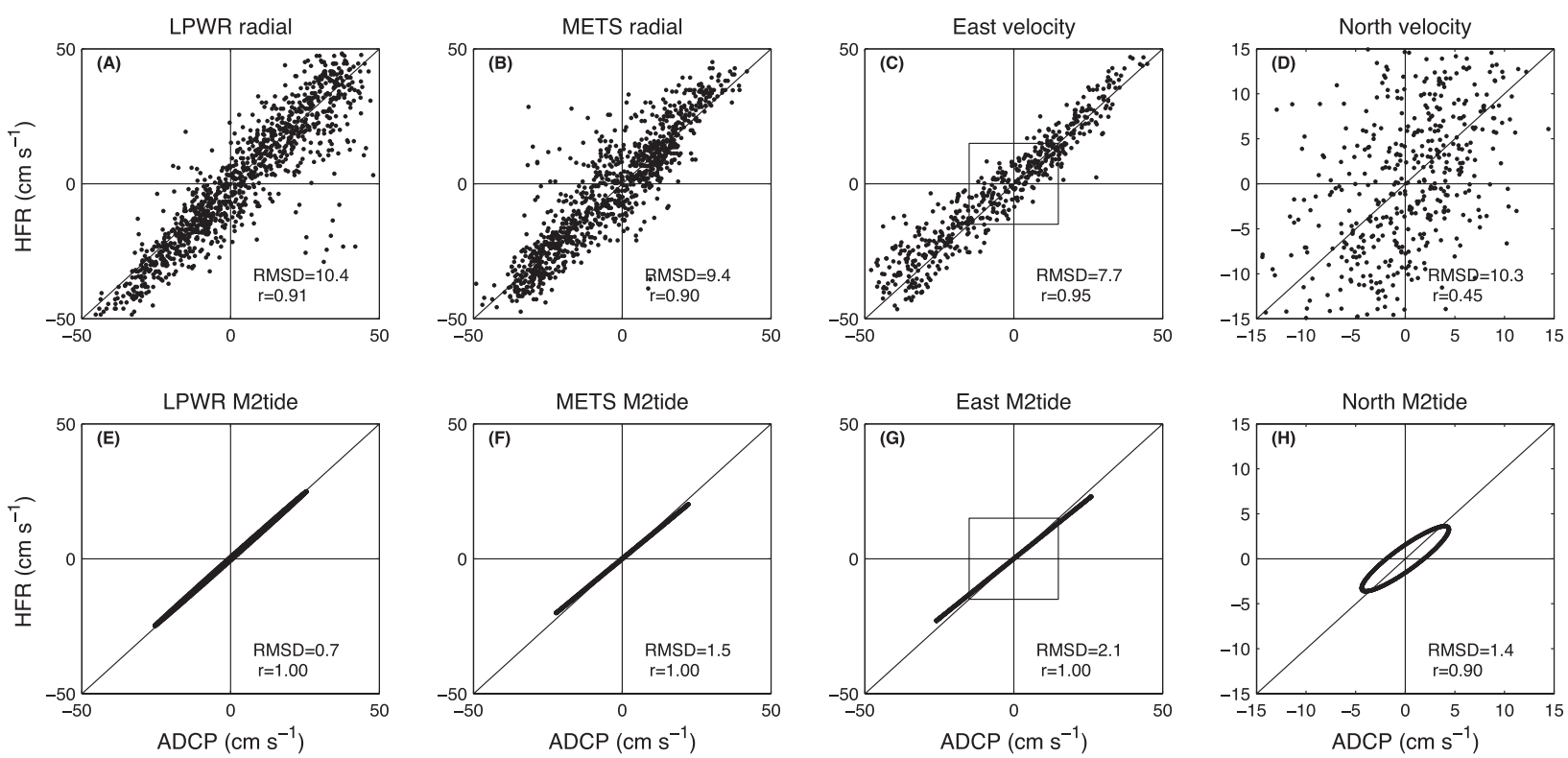

FIG. 4. Comparisons of HFR radial and vector surface currents measured by the LPWR and METS sites with in situ velocities from the node ADCP for the fall dataset. Both (a)-(d) hourly averaged data and (e)-(h) estimated $M_{2}$ tidal components are shown. The scale of the smaller axis used for the north results $(\mathrm{d}),(\mathrm{h})$ is illustrated in the east results $(\mathrm{c}),(\mathrm{g})$ (square box).

of the $M_{2}$ tidal component of the radial velocities had rms differences of 0.7 and $1.5 \mathrm{~cm} \mathrm{~s}^{-1}$ for LPWR and METS, respectively. Rms differences for the $M_{2}$ tidal component of the vector velocities were larger at 2.1 and $1.4 \mathrm{~cm} \mathrm{~s}^{-1}$ for the east and north components, but showed a significant phase lag for the smaller northward tidal component (Fig. 4). For the winter dataset, rms differences between the $M_{2}$ tidal component of radial surface currents varied from 0.2 to $2.2 \mathrm{~cm} \mathrm{~s}^{-1}$ for the three sites while rms differences for the east and north components were and 1.3 and $1.8 \mathrm{~cm} \mathrm{~s}^{-1}$, respectively (Fig. 5). As seen in the fall dataset, the smaller northward tidal component exhibited a significant phase offset from the ADCP-based tide.

These initial results show that the MVCO system has noise levels that were equal to or exceed the typical
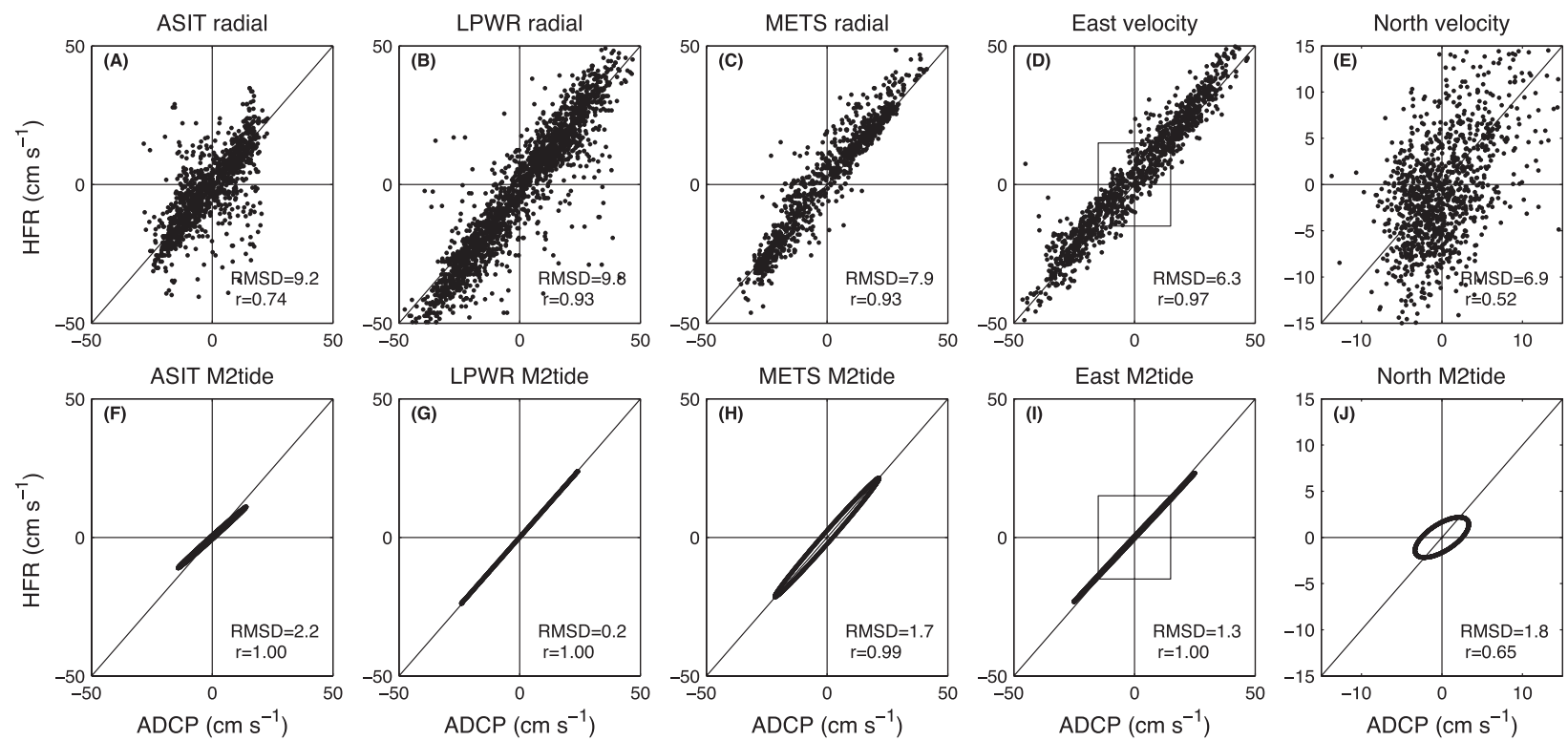

FIG. 5. Comparisons of HFR radial surface currents measured by all three radial sites: (a),(f) ASIT, (b),(g) LPWR, (c),(h) METS as well as the (d),(i) east, and (e),(j) north vector components. See Fig. 4 for detailed descriptions. 
uncertainty levels given above. Further tests, not shown here, indicated that the addition of the third site led to much of the reduced uncertainty levels seen in the vector surface currents of the winter dataset. However, neither dataset was able to produce an unbiased estimate of the northward, across-shelf component of the $M_{2}$ tide. Because the across-shelf component of the tide is of the same magnitude as the dynamically important across-shelf surface velocities that are critical to understanding across-shelf exchange, additional analysis methods are necessary to reduce these observed biases as well as noise levels in general.

\section{Additional data quality controls}

Previous efforts to reduce errors in radial velocity estimates have generally focused on the instrument configuration causes of velocity errors, including incorrect firstorder delineations, beam patterns, or incorrect MUSIC parameters (Emery et al. 2004; Kohut and Glenn 2003; de Paolo and Terrill 2007a). As described above, a significant effort was made to optimize these user-controllable instrument parameters for the MVCO HFR system. To extract additional error reductions from the datasets, the following two approaches were investigated: i) using the radial metric output of the CODAR Ocean Systems' processing software to develop nonvelocity-based data quality control thresholds, and ii) implementing a new way to selectively average radials falling within the same cell to account for differences in data quality.

\section{a. Data quality thresholds}

For a given range circle and radial velocity identified in the cross-spectra of the received signal (see Fig. 1) the complex signal amplitudes from the three antennas and the measured antenna response pattern are used by MUSIC to estimate the DOA function (Fig. 6). The updated software release (version 7) produces additional output, called radial metrics, that give details about the signal properties and direction-finding calculation, including DOA peak power $(\mathrm{dB})$, DOA half-power width $\left(^{\circ}\right)$, estimated true signal power $(\mathrm{dB})$, and SNR values from each antenna element. These metrics were examined using the observations collected by all MVCO radar sites to develop quality control thresholds based on the assumption that sharper DOA functions with higher SNRs are indicative of results with reduced potential errors. While a range of metric combinations and threshold levels were examined here, the following thresholds were found to be a reasonable balance of data quality verses data quantity: (a) the DOA peak power greater than $5 \mathrm{~dB}$, (b) DOA half-power width less than $50^{\circ}$, and (c) the SNR in the monopole receive antenna (antenna 3)
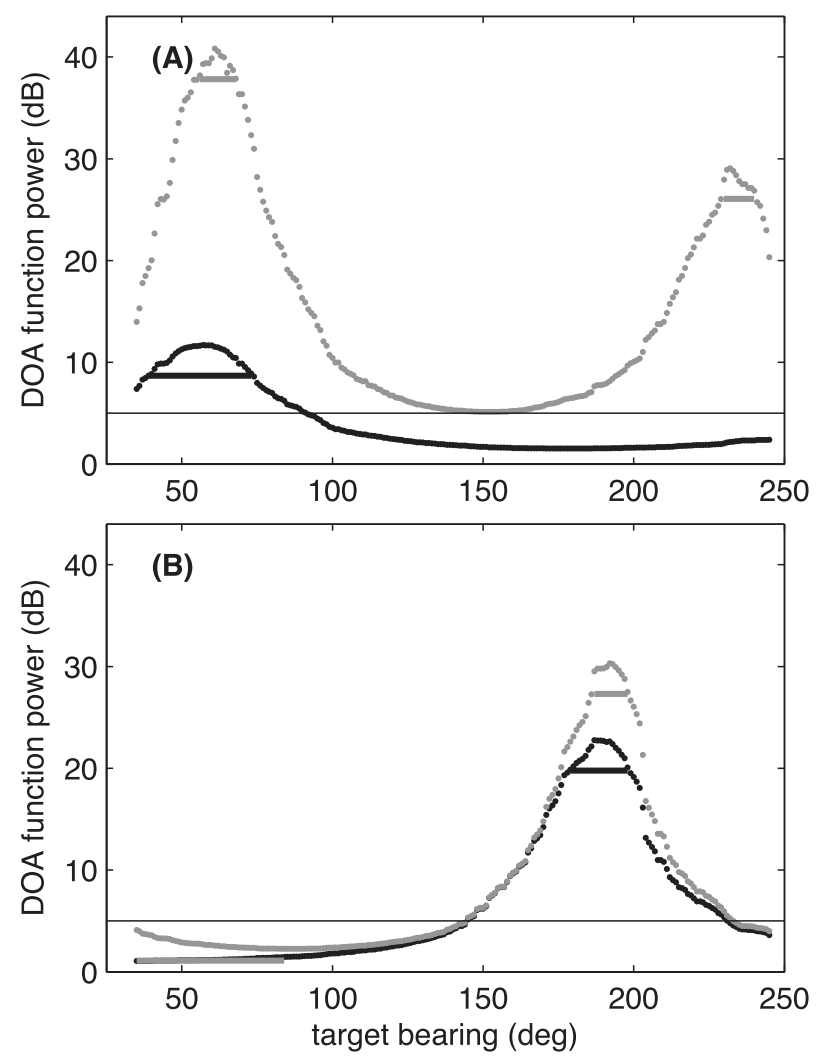

FIG. 6. Two examples of the DOA functions estimated by MUSIC for different range-velocity combinations of the cospectra collected by METS at 0045 UTC 15 Dec 2010. The single- (black) and dual- (gray) angle estimates of the DOA function are shown, along with the DOA peak power (thin lines at $5 \mathrm{~dB}$ ) and halfpower width (thick lines) thresholds described in the text. For these examples, the dual-angle solution parameters used by COS processing software chose the dual-angle solution for example (a) and the single-angle solution for example (b).

greater than 5 . For each velocity inside the first-order region considered, unaveraged radials with results falling below the thresholds given were eliminated before computing spatial and temporal radial averages. The effects of these thresholds on data distribution can be illustrated by examining the ratios of radials falling below each threshold to those passing all thresholds, as shown for the winter dataset at LPWR in Fig. 7. In general, application of these threshold criteria have the strongest effects in areas where data are likely erroneous, such as over land or at the extremities of the coverage area. However, the half-power width threshold appears to eliminate additional raw radials throughout the domain. The effects of this combination of data quality thresholds on the results are examined below.

\section{b. Weighted averaging}

A second path to improved data quality focuses on the representativeness of individual radial velocity estimates. 


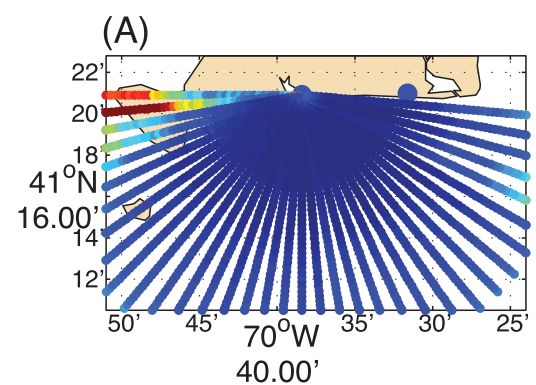

(B)

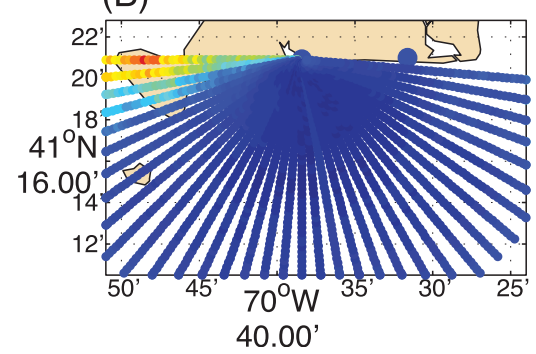

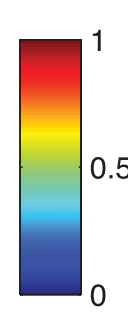

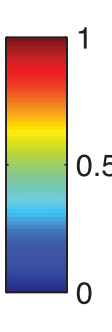

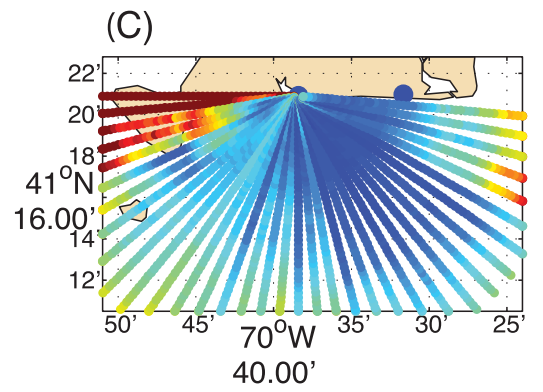
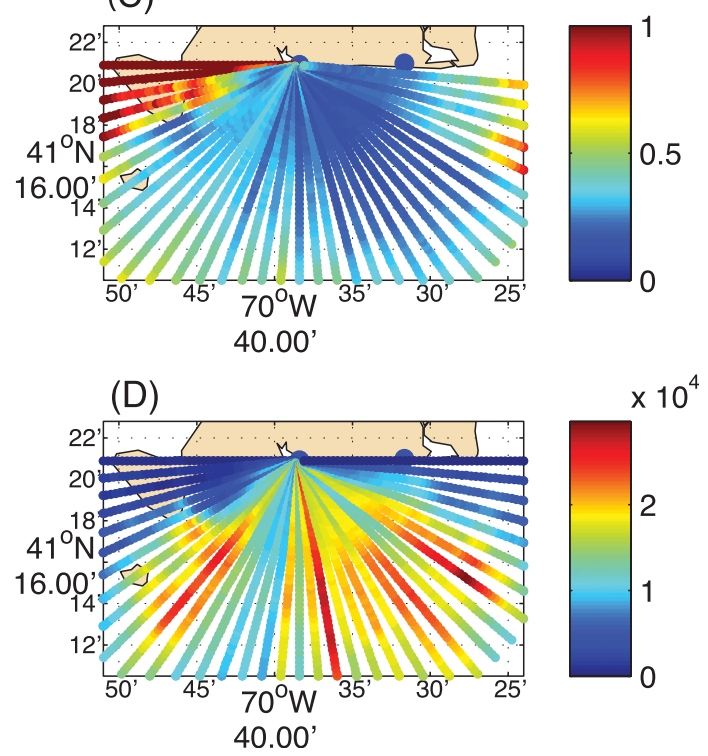

FIG. 7. Spatial maps of the data discarded and retained after application of the three quality control thresholds on the LPWR winter dataset. The fraction of radial velocities discarded by each of the threshold criteria: (a) DOA peak value less than $5 \mathrm{~dB}$, (b) antenna-3 SNR less than $5 \mathrm{~dB}$, or (c) DOA half-power peak width greater than $50^{\circ}$ are given for each radial position. (d) The failure rates of each threshold are normalized (color scale $0-1$ ) by the total number of valid radials passing all thresholds.

As described above, all radials along a range circle with estimated bearings falling within a nominally $5^{\circ}$ azimuthal range are averaged to produce the final radial estimate. Weighting the raw radial results in a given range/azimuthal cell by the output metrics of MUSIC used above was explored as a way to reduce the noise and potential bias of the radial averages. The SNR from antenna 3 (the monopole) and the MUSIC-based estimate of the true signal power (as a complex signal voltage) appeared to be the most potentially useful weighting functions. Weighting the radial averages by additional radial metrics such as DOA peak power or DOA half-power width were investigated, but the antenna-3 SNR and the signal power weights had both the most reasonable physical interpretation as well as the largest impact on the radial averages.

Assuming that the wind forcing over an individual range/azimuthal cell is constant, or nearly constant, a radial velocity estimate having a true signal power larger than that of a second radial from the same range/azimuthal cell can be interpreted as being more representative of the true surface velocity over the cell's area. Physically, this can be thought of as more of the cell "moving" at the first velocity; thus, more individual Bragg waves are Doppler shifted by this first velocity, leading to a stronger return from the cell at that velocity. An example of a situation where weighting the average this way is significantly different from a simple average is shown in Fig. 8. Here, velocities varying from -30 to $15 \mathrm{~cm} \mathrm{~s}^{-1}$ have estimated bearings that fall within the cell domain. However, the more positive velocities have reduced signal power relative to the more negative velocities (Fig. 8). In this example, which is typical of the results from the MVCO system, the estimated true signal power appears to have a larger dynamic range than the antenna-3 SNR. Where an arithmetic mean of the six velocities gives a value of $-13 \mathrm{~cm} \mathrm{~s}^{-1}$, weighting the individual radials by their MUSIC-estimated signal power (as a voltage) gives $-25 \mathrm{~cm} \mathrm{~s}^{-1}$, lessening the impact of these weaker power velocity estimates. Because of its larger impact, generally found to be true for all sites of both datasets, the true signal power is used below as the weighting function.

\section{c. Application to the MVCO HFR system}

To understand the relative value of the additional quality control measures proposed here, HFR-based surface currents estimated using these methodologies were also compared to the ADCP-based surface velocities from the MVCO 12-m node. Specifically, radial velocities were computed for the two datasets using the following three data quality control methods:

- Standard: Use the standard SeaSonde data processing steps for MVCO HFR datasets as described in section 3. Summarized here again for clarity: from the ensemble-averaged spectra (composed of 25-min 

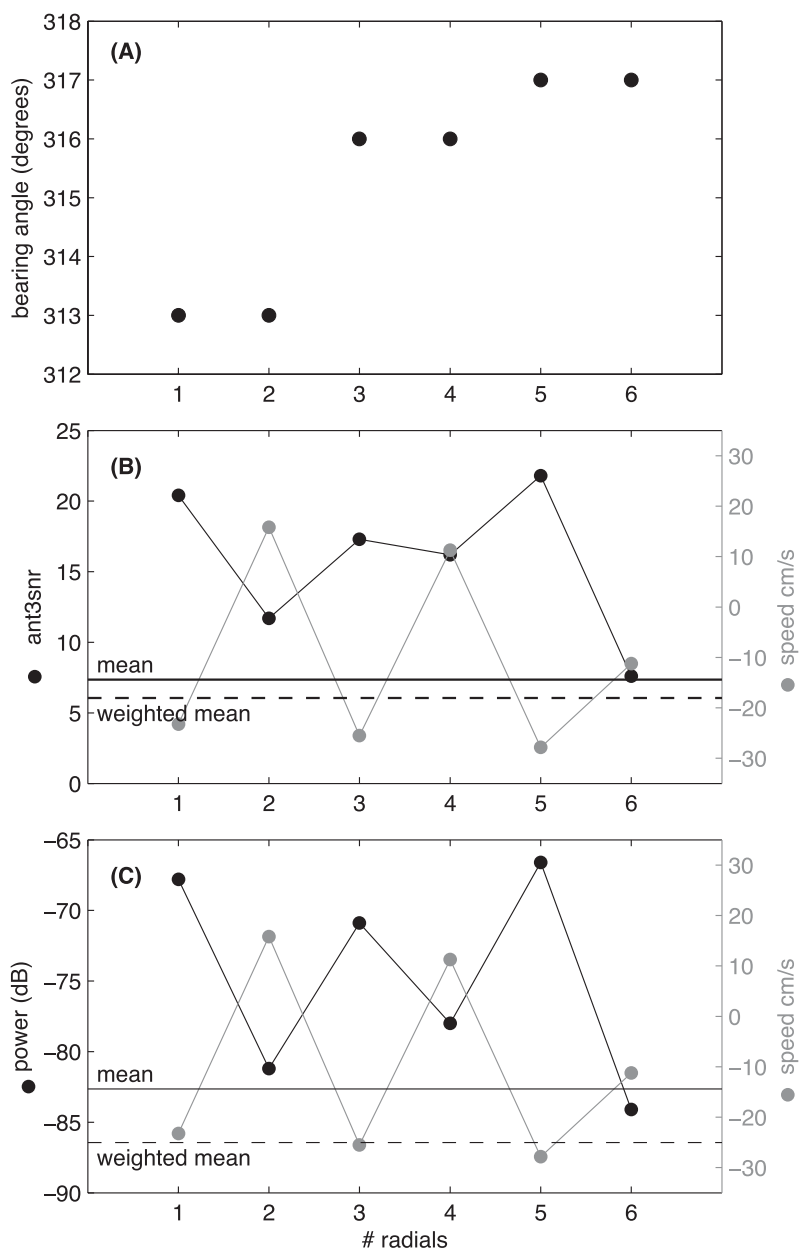

FIG. 8. The effect of using arithmetic verses power-weighted spatial averaging to condense the full MUSIC results to $5^{\circ}$ azimuthal bins. Shown are (a) the bearing angle as well as the (b) antenna-3 SNR and (c) signal power (both with speed overlain) for six radial velocities falling within the same azimuthal bin along range cell 20 of ASIT at 1145 UTC 5 Dec 2010. The arithmetic mean of the six radials [shown by the black line in (b) and (c)] was $-13 \mathrm{~cm} \mathrm{~s}^{-1}$, while the antenna-3 SNR weighted mean was $-17 \mathrm{~cm} \mathrm{~s}^{-1}$ [dashed line in (b)]. Because the more positive radial speed had much weaker signal power, the power-weighted average was $-25 \mathrm{~cm} \mathrm{~s}^{-1}$ [dashed line in (c)].

average every $15 \mathrm{~min}$ ), estimate bearings via MUSIC and spatially average results into $5^{\circ}$ azimuthal radial averages.

- Thresholds: Use the radial metric output from the ensemble-averaged spectra to exclude radial velocities from the spatial averages, and the subsequent vector surface currents, when one of the following is satisfied: (a) the DOA peak power $<5 \mathrm{~dB}$, (b) DOA half-power width $>50^{\circ}$, or (c) antenna-3 SNR $<5$, before spatial averaging to the standard radial grid for each site.

- Thresholds/weights: Use the radial metric output from the ensemble-averaged spectra and apply the threshold criteria described above before computing power-weighted spatial averages on the standard radial grid.

\section{Methodological comparisons}

For each of the processing methods defined above, vector velocities were formed for each grid point of an identical grid from the available radial velocities. Both the radial and vector velocity estimates from the HFR, as well as the ADCP, were hourly averaged to a common time stamp for comparison. The rms differences and correlation coefficients between the HFR and 12-m-node ADCP surface velocities for each of these three methodologies are given here for both radial and vector velocities from the fall (Fig. 9) and winter (Fig. 10) datasets.

For the majority of the radial velocity comparisons, rms differences decreased and correlations increased between the "Standard," "Thresholds," and "Thresholds/ weights" results. For the fall dataset, the new quality controls had the most effect at METS (Fig. 9). Rms differences between the ADCP and METS radials for the thresholds/weights method were $7 \mathrm{~cm} \mathrm{~s}^{-1}$, which was a decrease of $2.5 \mathrm{~cm} \mathrm{~s}^{-1}$ over the standard method. Rms differences for LPWR verses the ADCP decreased from 1.1 to $9.4 \mathrm{~cm} \mathrm{~s}^{-1}$ between the standard and thresholds/ weights results. Rms differences for the estimated $M_{2}$ tidal constituent during the fall dataset decreased slightly for the comparison using LPWR radials, but decreased more than $1.25 \mathrm{~cm} \mathrm{~s}^{-1}$ between the standard and thresholds/weights methods for METS. For the hourly averaged radial velocities of the winter dataset, rms differences at ASIT and METS either increased or showed no change (Fig. 10), although rms differences for LPWR decreased $1 \mathrm{~cm} \mathrm{~s}^{-1}$ with the additional quality controls. In contrast to the full dataset, comparisons of the estimated $M_{2}$ tidal constituents actually improved by $1 \mathrm{~cm} \mathrm{~s}^{-1}$ for ASIT and $0.5 \mathrm{~cm} \mathrm{~s}^{-1}$ for METS with the additional methods, but degraded slightly at LPWR.

For both the east and north components, rms differences in the velocity comparisons decreased as additional data quality measures were utilized. During the fall dataset, rms differences improved from 1 to $6.7 \mathrm{~cm} \mathrm{~s}^{-1}$ for east velocities and from 2 to $8.3 \mathrm{~cm} \mathrm{~s}^{-1}$ for north velocities between the standard and thresholds/weights methods, with correlations increasing as well (Fig. 9). Comparisons of the estimated $M_{2}$ component of the east and north tidal velocities showed decreases in the rms differences of more than $1 \mathrm{~cm} \mathrm{~s}^{-1}$ between the standard method and those with additional quality controls. Application of the threshold criteria alone appear to make the most difference for the fall east velocity component, because the 

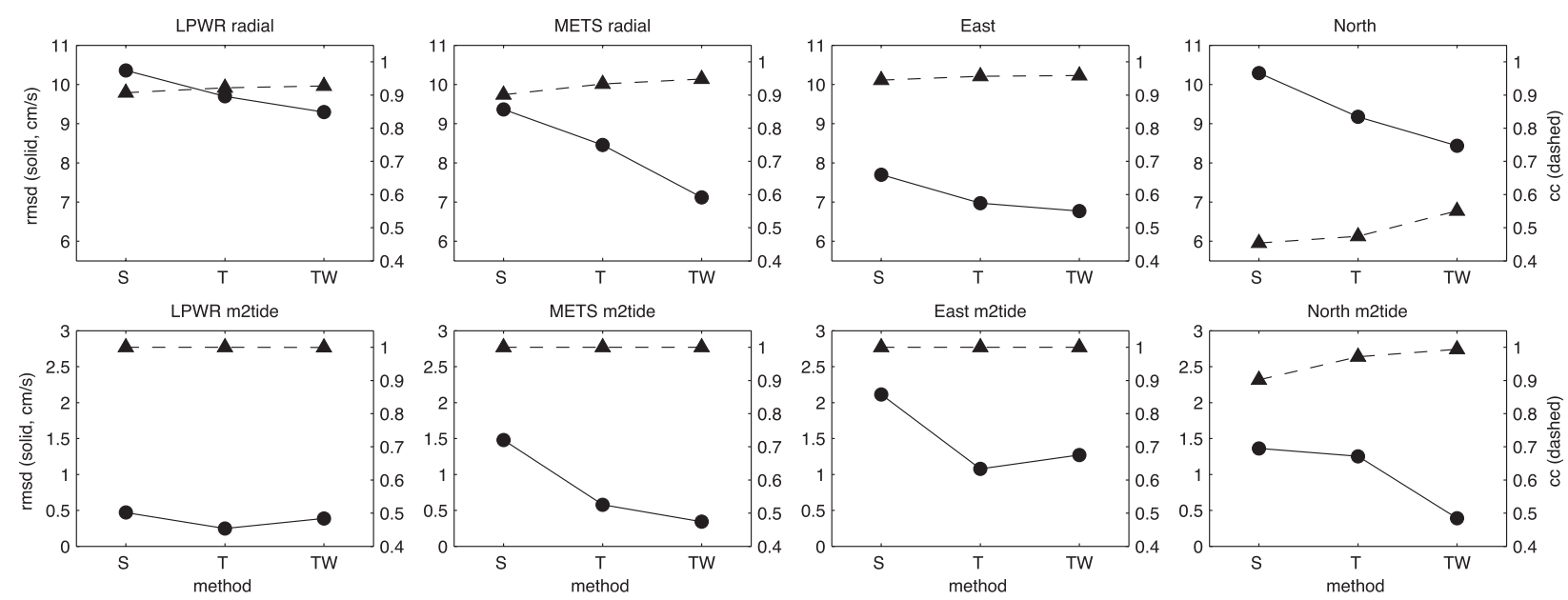

FIG. 9. Comparison of the rms differences and correlation coefficients between the ADCP and LPWR and METS HFR velocity estimates made with the standard $(S)$, thresholds $(T)$, and thresholds/weights (TW) methods applied to the fall dataset shown in Fig. 4. The rms differences ( $\mathrm{cm} \mathrm{s}^{-1}$; circles and solid lines, left-hand axes) and correlation coefficients (triangles and dashed lines, right-hand axes) are shown in each panel for the three HFR processing methods. Results are shown for (top) raw velocites and (bottom) $M_{2}$ tidal fits for both radial velocities (from left to right) from each site and combined east and north velocities.

decrease for the thresholds method is greater than that of the combined thresholds/weights method. However, for the smaller northward tidal component, the addition of the weighted average was critical to realizing the decrease in rms difference seen, because the largest decrease occurs for that method only (Fig. 9). For the winter dataset, east hourly rms differences decreased by 0.75 to $5.75 \mathrm{~cm} \mathrm{~s}^{-1}$ from the standard to the thresholds/weights methods, while the north rms differences decreased by 0.5 to $7.5 \mathrm{~cm} \mathrm{~s}^{-1}$ (Fig. 10). Comparisons of the east and north estimated $M_{2}$ tidal components for the winter dataset showed up to a $1 \mathrm{~cm} \mathrm{~s}^{-1}$ decrease in rms values from the standard method. In both cases, application of the thresholding criteria appeared to account for the largest part of the decreased rms difference realized.
The maximum reduction in $\mathrm{rms}$ difference seen, $2.5 \mathrm{~cm} \mathrm{~s}^{-1}$, represents a significant $(50 \%)$ drop in the error variance, based on the total rms difference values for vector velocities. Additionally, application of the new methods had an important effect on minimizing the observed biases of the $M_{2}$ tidal components. As described above, a biased estimate of a narrowband signal such as the $M_{2}$ tide is an indicator of additional data quality problems within the dataset. However, the additional quality controls were able to reduce the phase difference between the ADCP and HFR results for both datasets (Fig. 11). In the fall period, only the thresholds/ weights data product was able to match the phase and approximate magnitude of the $4 \mathrm{~cm} \mathrm{~s}^{-1}$ amplitude northern component of the $M_{2}$ tide. For the weaker tide
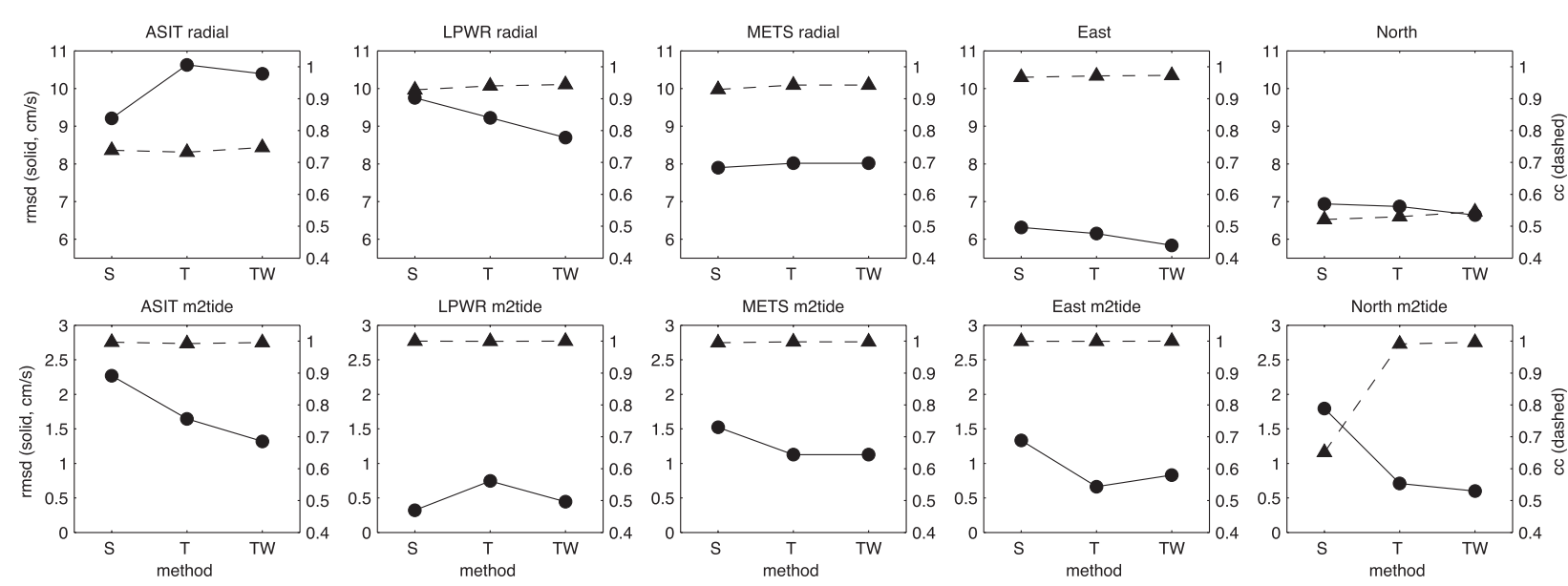

FIG. 10. As in Fig. 9, but for the winter dataset, which included data from the third site, ASIT. 


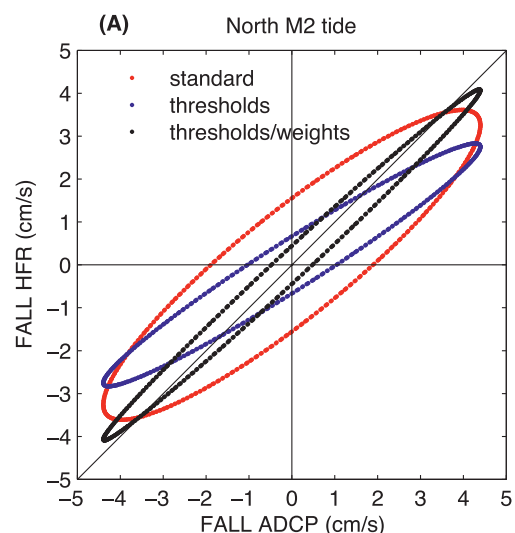

(B)

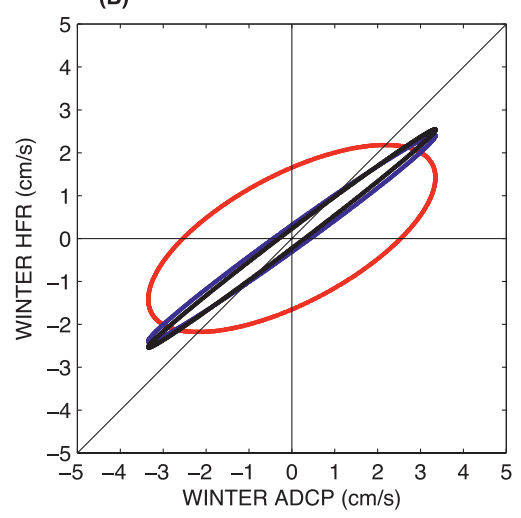

FIG. 11. Scatterplots of the north component of the $M_{2}$ tidal fit from all methods for the (a) fall and (b) winter data periods.

present during the winter period, both advanced methods were closer in phase to the ADCP estimate than the standard method (Fig. 11), but are $0.8 \mathrm{~cm} \mathrm{~s}^{-1}$ smaller in magnitude than the ADCP-estimated tidal magnitude.

The 12-m node location was not the only location within the MVCO HFR domain having significant changes in the magnitude of the $M_{2}$ tide between the data products. Figure 12 shows the spatial structure of the $M_{2}$ tidal ellipses during the winter data period (top), using the thresholds/weights method, as well as the differences between the semimajor and semiminor axes of standard and thresholds/weights methods (bottom). In the lower panel, changes in the semimajor (semiminor) axis are plotted along the east-west (north-south) axis, with positive changes being eastward (northward). Thus, eastward and northward changes represent increases in axis magnitudes between the standard and thresholds/ weights methods. Differences between the two products vary spatially. In the region around ASIT both major and minor axes are $2-4 \mathrm{~cm} \mathrm{~s}^{-1}$ greater using the thresholds/ weights methods, meaning that the advanced data product is able to remove more of the tidal energy present. Farther offshore, the major axis increases are often larger than the changes seen to the minor axis.
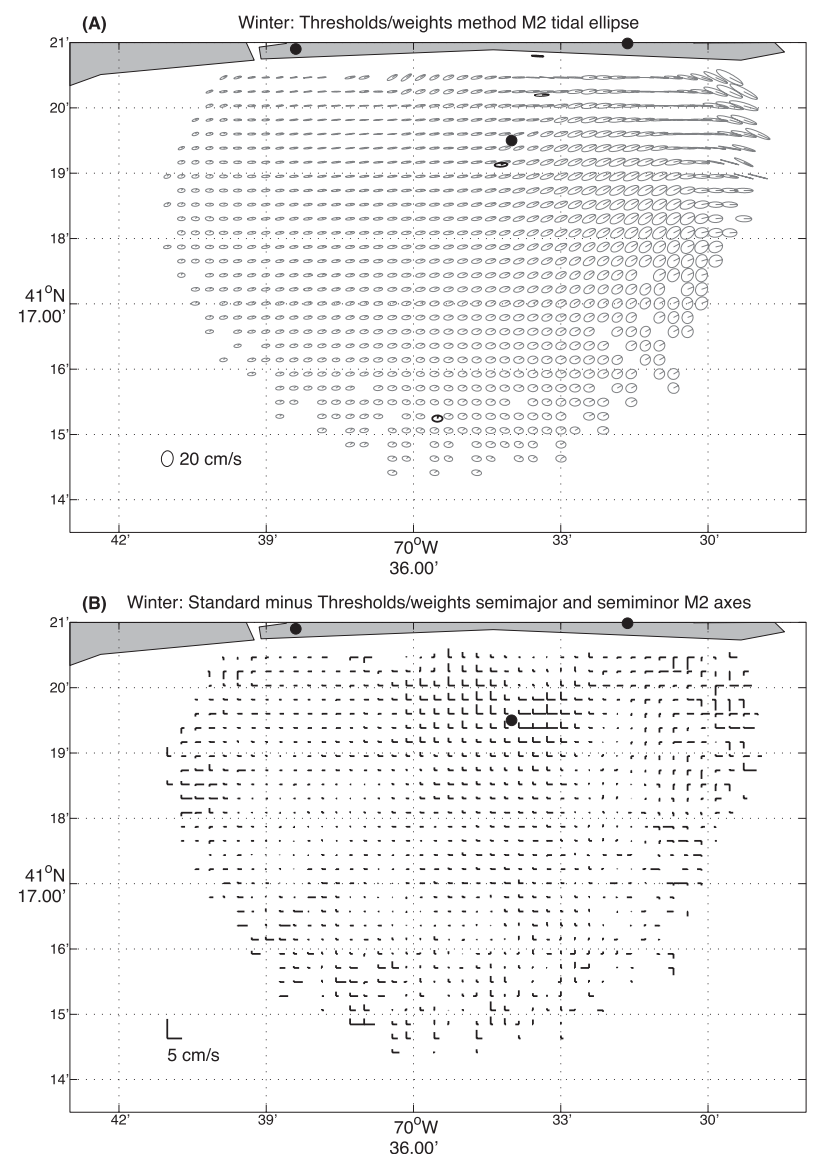

FIG. 12. (a) Spatial map of the $M_{2}$ tidal ellipses for the thresholds/ weights method results. (b) Differences of the semimajor and semiminor axis of the thresholds/weights method from the standard method results. Eastward and northward changes represent increases in axes magnitude between standard and thresholds/weights.

In addition to thresholds/weights $M_{2}$ tidal ellipses agreeing more closely with results from the 12 -m node ADCP, tidal ellipses using this method were more similar to ellipses from a recently deployed array of ADCP measurements (Lentz et al. 2008) at 7-, 17-, and 27-m water depths (also shown in black in the upper panel of Fig. 12). The ADCP estimates are based on measurements obtained during the same calendar dates as the HFR winter dataset, but in 2008-09. While these additional comparisons are not as robust as the direct comparisons with the 12-m node ADCP, they do offer additional evidence that the thresholds/weights methods offer a better estimate over a wide region of the MVCO domain.

\section{Conclusions}

This work has illustrated how a number of changes to the standard processing methods utilized to derive surface 
currents from HFR measurements can further reduce velocity differences with in situ observations. Both the inclusion of minimum threshold criteria for the received signal parameters and direction-finding metrics as well as the use of weighted radial velocity averages were needed to provide the maximum reductions seen. Importantly, both strategies have ready physical interpretations. The threshold criteria used eliminated radial estimates where the DOA function was poorly defined; thus, the radials would have high bearing uncertainty. The same was true for radials where the signal-to-noise ratio of the monopole antenna (antenna 3) was small. Weighting the velocity averages by the MUSIC-estimated power level gives the stronger returns, considered to be more representative of the velocity within the averaging cell, which increased significance. Both the threshold and weighting strategies also have the potential to be easily integrated into the standard processing software in a way that would allow for user controls of the parameters of interest.

Specific to the newly deployed MVCO HFR array, application of these methods reduced the errors between HFR and in situ sensors by up to $2 \mathrm{~cm} \mathrm{~s}^{-1}$ for hourly estimates of north and east velocities. However, as is evident from the smaller rms differences for the hourly estimates of the winter dataset compared to the fall dataset, the increase in accuracy resulting from the advanced data quality methods was exceeded by the increase resulting from the addition of a third radar site in the middle of the domain. The addition of this site enabled decreased rms differences with the 12 -m node ADCP to values approaching $5.5 \mathrm{~cm} \mathrm{~s}^{-1}$ using the advanced methods. Based on literature estimates, these discrepancies are at or near the noise floor (instrument + geophysical) for the instruments, especially given the increased independence of the radial and vector estimates obtained.

However, it is important to note that the addition of the third site did not alter the bias of the $M_{2}$ tidal constituents seen in the standard method results from either dataset. Application of the advanced quality controls described here were necessary to reduce the observed biases of this basic component of the tidal velocity. Again, the ability of HFR-based surface currents to resolve a narrowband signal, such as the $M_{2}$ tide, without significant bias is important for multiple reasons. With a biased estimate of the tide, the true tidal signal cannot be properly removed for analyses of synoptic events or subtidal dynamics. Additionally, a biased tidal signal is a likely indicator for similar biases in the nontidal velocities themselves. Thus, addition of these advanced data quality methods is seen as being especially critical to making unbiased observations of the smaller, across-shelf components of surface velocity.
Acknowledgments. The authors thank CODAR Ocean Systems for providing advance copies of their updated Radial Site Software Suite and D. Barrick for helpful discussions. Additionally, J. T. Farrar, D. Fratantoni, and L. Washburn, as well as two anonymous reviewers, provided helpful comments on the manuscript. The development and operation of the MVCO HFR system was supported by the Woods Hole Oceanographic Institution.

\section{REFERENCES}

Barrick, D. E., and B. L. Weber, 1977: On the nonlinear theory for gravity waves on the ocean's surface. Part II: Interpretation and application. J. Phys. Oceanogr., 7, 11-21.

_ terns in CODAR ocean surface measurements. IEEE J. Oceanic Eng., 11, 304-309.

$\longrightarrow$, and - 1997: Evolution of bearing determination in HF current mapping radars. Oceanography, 10, 72-75.

COS, 2009: SeaSonde Radial Site Software Suite documentation, release 6. Codar Ocean Systems.

Cosoli, S., A. Mazzoldi, and G. Miroslav, 2010: Validation of surface current measurements in the northern Adriatic Sea from highfrequency radars. J. Atmos. Oceanic Technol., 27, 908-919.

Creamer, D., F. Henyey, R. Schult, and J. Wright, 1989: Improved linear representation of ocean surface waves. J. Fluid Mech., 205, 135-161.

Crombie, D., 1955: Doppler spectrum of the sea echo at $13.56 \mathrm{Mc} / \mathrm{s}$. Nature, 175, 681-682.

de Paolo, T., and E. Terrill, 2007a: Properties of HF radar compact antenna arrays and their effect on the MUSIC algorithm. Scripps Institution of Oceanography Tech. Rep., 40 pp. [Available online at http://escholarship.org/uc/item/5bw303tj.]

_ and — 2007b: Skill assessment of resolving ocean surface current structure using compact-antenna style HF RADAR and the MUSIC direction finding algorithm. J. Atmos. Oceanic Technol., 24, 1277-1300.

Emery, B., L. Washburn, and J. Harlan, 2004: Evaluating radial current measurements from CODAR high-frequency radars with moored current meters. J. Atmos. Oceanic Technol., 21, 1259-1271.

Ganju, N. K., S. J. Lentz, A. R. Kirincich, and J. Farrar, 2011: Complex mean circulation over the inner shelf south of Martha's Vineyard revealed by observations and a high-resolution model. J. Geophys. Res., 116, C10036, doi:10.1029/2011JC007035.

Halle, C., 2008: HF radar processing using "nearest-neighbor" statistics. Bodega Marine Laboratory Tech. Rep., 26 pp. [Available online at http://bml.ucdavis.edu/boon/pdf/Methods PaperHalle.pdf.]

Kim, S. Y., E. Terrill, and B. Cornuelle, 2007: Objectively mapping $\mathrm{HF}$ radar-derived surface current data using measured and idealized data covariance matrices. J. Geophys. Res., 112, C06021, doi:10.1029/2006JC003756.

Kohut, J. T., and S. Glenn, 2003: Improving HF radar surface current measurements with measured antenna beam patterns. J. Atmos. Oceanic Technol., 20, 1303-1316.

_ H. J. Roarty, and S. M. Glenn, 2006: Characterizing observed environmental variability with HF Doppler radar surface current mappers and acoustic Doppler current profilers: Environmental variability in the coastal ocean. IEEE J. Oceanic Eng., 31, 876-884, doi:10.1109/JOE.2006.886095. 
Laws, K., J. Paduan, and J. Vesecky, 2010: Estimation and assessment of errors related to antenna pattern distortion in CODAR seasonde high-frequency radar ocean current measurements. J. Atmos. Oceanic Technol., 27, 1029-1043.

Lentz, S., M. Fewings, P. Howd, J. Fredericks, and K. Hathaway, 2008: Observations and a model of undertow over the inner continental shelf. J. Phys. Oceanogr., 38, 2341-2357.

Lipa, B. J., B. Nyden, D. Ullman, and E. Terrill, 2006: Seasonde radial velocities: Derivation and internal consistency. IEEE J. Oceanic Eng., 31, 850-861.

Mao, Y., and M. Heron, 2008: The influence of fetch on the response of surface currents to wind studied by the HF Ocean Surface Radar. J. Phys. Oceanogr., 38, 1107-1121.

NDBC, 2009: Handbook of automated data quality control checks and procedures. NDBC Tech. Doc. 09-02, 78 pp. [Available online at http://www.ndbc.noaa.gov/NDBCHand bookofAutomatedDataQualityControl2009.pdf.]

Ohlmann, C., P. White, L. Washburn, E. Terrill, B. Emery, and M. Otero, 2007: Interpretation of coastal HF radar-derived surface currents with high-resolution drifter data. J. Atmos. Oceanic Technol., 24, 666-680.
Paduan, J., and H. Graber, 1997: Introduction to high-frequency radar: Reality and myth. Oceanography, 10, 36-39.

_ K. C. Kim, M. S. Cook, and F. P. Chavez, 2006: Calibration and validation of direction-finding high-frequency radar ocean surface current observations. IEEE J. Oceanic Eng., 31, 862875, doi:10.1109/JOE.2006.886195.

Pawlowicz, R., B. Beardsley, and S. Lentz, 2002: Classical tidal harmonic analysis including error estimates in MATLAB using T_TIDE. Comput. Geosci., 28, 929-937.

Schmidt, R., 1986: Multiple emitter location and signal parameter estimation. IEEE Trans. Antennas Propag., 34, 276-280.

Stewart, R., and J. Joy, 1974: HF radio measurements of surface currents. Deep-Sea Res., 21, 1039-1049.

Ullman, D. S., and D. L. Codiga, 2004: Seasonal variation of a coastal jet in the Long Island Sound outflow region based on HF radar and Doppler current observations. J. Geophys. Res., 109, C07S06, doi:10.1029/2002JC001660.

, J. O'Donnell, J. Kohut, T. Fake, and A. Allen, 2006: Trajectory prediction using HF radar surface currents: Monte Carlo simulations of prediction uncertainties. J. Geophys. Res., 111, C12005, doi:10.1029/2006JC003715. 